\title{
Effects of Previous Curare-Immobilization on Pavlovian Conditioned Heart Decelerations in the Curarized Rat
}

\author{
J. ROGER WILSON² AND LEO V. DICARA \\ Department of Psychiatry, Mental Health Research Institute, University of Michigan \\ Medical Center, The University of Michigan, Ann Arbor, Michigan 48104
}

(Received 28 December 1973)

\begin{abstract}
WILSON, J. R. AND L. V. DICARA. Effects of previous curare-immobilization on Pavlovian conditioned heart rate decelerations in the curarized rat. PHYSIOL. BEHAV. 14(3) 259-264, 1975. - Recent evidence indicates that mechanisms other than the vagal-blocking properties of d-tubocurarine chloride (dTC) or inadequate respiration may account for the reports of invariant heart rate (HR) in curarized rats. Two experiments were conducted to determine if repeated dTC-paralysis facilitate acquisition of a Pavlovian HR conditioning in the dTC-paralyzed rat. In Experiment 1, Pavlovian HR conditioning was examined in curarized rats which had (1) experience with dTC-induced immobilization before conditioning, or (2) no experience with dTC-induced immobilization. The results showed that pronounced conditioned HR decelerations were observed only for the curare-experienced animals. Experiment 2 was conducted to determine if repeated curarization influenced tolerance to dTC-induced neuromuscular paralysis. No group differences in basal electromyographic (EMG) activity or time to recover from paralysis were found between the Non-Curare Adapted (NCA), and the Curare Adapted (CA) groups. The implications of the results are discussed in terms of the role of immobilization-induced stress on acquiring stimulus control over autonomic responses.
\end{abstract}

Autonomic conditioning Heart rate Curariform drugs Electromyographic potentials Rats

THE problems in demonstrating operant $[3,4,14]$ and classical $[7,10,16,17]$ heart rate (HR) conditioning in the curarized animal have drawn attention to potential complications arising from the pharmacological consequences of neuromuscular paralysis and inadequate respiration. For instance, there is mounting evidence that preganglionic influences of d-tubocurarine chloride (dTC) may interfere with cardiac reactivity as well as skeletal responding. Black [1] and Galosy et al. [8] with dogs and cats, respectively, reported that systemic injections of dTC attenuated the cardiodeceleration produced by direct vagal stimulation. More recently, Dworkin [4] and Schwartz and Hahn [18] observed that pulmonary vascular edema and disturbances in acid-base balance often accompanied paralysis in rats when the ventilation parameters were similar to those used in earlier reports of operant HR conditioning. However, rats appear to be less sensitive to certain side-effects of paralysis than other species. For instance, Hahn and Slaughter [10] failed to observe conditioned HR decelerations in rats, but did report pronounced cardiac orienting responses in the form of HR decelerations that rapidly habituated. Moreover, Hahn [9] recently demonstrated that cardiodeceleration could be produced by direct vagal stimulation in curarized rats if precautions were taken to maintain normal respiration and body temperature.

Physical restraint or immobilization has been a conventional means of inducing a physiological stress response [22]. Since curare-paralysis would appear to be the most effective method of immobilizing an animal, it is interesting that curariform drugs have failed to attract adequate consideration as potent stress inducers. Accordingly, it might be anticipated that immobilization accompanying paralysis may itself contribute to the invariant HR observed with the curarized rat. For instance, Holdstock and Schwartzbaum [11] reported finding classical conditioning of HR decelerations in rats that were physically restrained by strapping the animal in a hammock-like apparatus. Each animal was permitted $2 \mathrm{hr}$ of adaptation to the restraining apparatus

${ }^{1}$ This research was supported by Grant No. MH 21403 from the United States Public Health Service, and No. $71-797$ from the American Heart Association to Leo V. DiCara. Portions of this study were presented at the Eastern Psychological Association Convention held in Philadelphia, Pennsylvania, 1974 and at the American Psychological Association Annual Meeting held in New Orleans, Louisiana, 1974. The authors gratefully acknowledge the technical advice of Mr. Ronald Derman.

${ }^{2}$ Address reprint requests to: J. Roger Wilson, Mental Health Research Institute, University of Michigan, Ann Arbor, Michigan 48104. 
one day before training began. Ray [16,17] and Wilson (unpublished observations) replicated this procedure but omitted the adaptation and found very small, if any, conditioned HR responses on the first day of conditioning. However, this performance improved with additional training sessions or with extended habituation sessions prior to conditioning.

Because of the possible nonspecific stress experienced during paralysis, curare adaptation might facilitate Pavlovian HR conditioning. Accordingly, the purpose of the following studies was to evaluate the influence of curare paralysis on Pavlovian HR conditioning in the curarized rat through the direct manipulation of curare-immobilization experience before conditioning. The influence of this experience on recovery from neuromuscular paralysis was also examined.

\section{EXPERIMENT 1}

METHOD

\section{Animals}

Twenty-four Sprague-Dawley albino rats purchased from Spartan Research Animals, Haslett, Michigan were used. All animals weighed $375-450 \mathrm{~g}$ when used in the experiment and had no previous experience with drugs. Each animal was housed individually and had free access to food and water for the duration of the experiment. The lights in the animal room were on from 8:00 a.m. to 8:00 p.m.

\section{Procedure}

Curare adaptation. Twelve of the animals were mildly etherized, curarized, and artificially respirated until the animal could breath without the aid of the respirator. These animals served in the Adaptation group. The adaptation procedure involved a single intraperitoneal $2.5 \mathrm{mg} / \mathrm{kg}$ injection of $\mathrm{dTC}(3.0 \mathrm{mg} / \mathrm{cc})$ after mild etherization. When the animal showed signs of respiratory distress, it was placed into a face mask modified from a rubber balloon [2]. The balloon was secured into place by placing the lower lip behind the upper incisors, and by pulling the upper lip tightly over the rat's mouth. The balloon was attached to a rubber stopper which held a Y-tube connected by a polyethylene tubing to the inspirator and expirator tube on a small animal respirator (Harvard 680). The animals were respirated at a $1: 1 \mathrm{I} / \mathrm{E}$ ratio at approximately $68-72 \mathrm{cpm}$ with a $6 \mathrm{cc}$ positive pressure volume/stroke. During immobilization, the animals lay on a folded towel and were respirated until they could regain sufficient motor control to sustain respiration without aid, and they were returned to their home cage. This procedure was repeated for each of the 3 consecutive days. At this point, the Pavlovian training phase was initiated. The remaining 12 animals were etherized, but not curarized and returned to their home cages. These animals made up the Nonadaptation group.

Pavlovian conditioning. In accordance with a $2 \times 2$ factorial design, half $(N=6)$ of the animals in the Adaptation group, and half $(\mathrm{N}=6)$ in the Nonadaptation group were trained in a Pavlovian HR conditioning paradigm following the 3 day adaptation phase of the experiment. The conditioning procedure was administered in three, 35-trial sessions distributed over 3 consecutive days. All animals were curarized in the same manner as used during adaptation. The procedure was as follows: After a $30 \mathrm{~min}$ curare stabilization period, 35 paired presentations of a conditioned stimulus (CS) and unconditioned stimulus (UCS) were introduced to half $(\mathrm{N}=6)$ of the animals in both the Adaptation and Nonadaptation groups. These were referred to as the Paired-Adapted (P-A) and PairedNonadapted (P-NA) groups, respectively. The remaining half of the animals in the Adaptation and Nonadaptation groups served as explicitly Unpaired sensitization control animals. These were referred to as the Unpaired-Adapted (UP-A) and the Unpaired-Nonadapted (UP-NA) groups, respectively. Within each session, these control animals received $35 \mathrm{CS}$-alone and $35 \mathrm{UCS}$-alone trials presented such that the interval between the CS termination and the UCS onset varied semi-randomly from 10 to $60 \mathrm{sec}$.

The CS consisted of a $51 / 2 \mathrm{sec}$ presentation of a $1 \mathrm{KHz}$ $80 \mathrm{db}$ (SPL) tone and $5 \mathrm{~W}$ light positioned in front of the animal. This CS was against a background of a $65 \mathrm{db}$ noise generated by the housing ventilation fan and coterminated with a $300 \mathrm{msec}, 1.2 \mathrm{~mA}$ RMS tail-shock (UCS). The tailshock was of a series resistance regulated variety that offered a relatively constant $60 \mathrm{~Hz}$ AC current. External relay switching controlled the line voltage at the primary of a 6:1 step-up transformer. The secondary was connected to the tail electrode through a voltage divider network employing a $200 \mathrm{~K} \Omega$ variable calibration resistor, a $200 \mathrm{~K} \Omega$ ten turn potentiometer, and a $300 \mathrm{~K} \Omega$ output resistor. High voltage switching transients were eliminated by the placement of a capacitor in parallel with the shock output.

The shocker was calibrated by placing a $1 \mathrm{~K} \Omega 1 \%$ resistor in series with the shock output while a $25 \mathrm{~K} \Omega$ was substituted for the tail. The differential inputs of a Tektronix oscilloscope were used to measure the voltage drop across the $1 \mathrm{~K} \Omega$ resistor and the current was then calculated from this value. The shock source was calibrated to give an output of $0.0-2.0 \mathrm{~mA}$ RMS. Variations in tail resistance within the expected range produced variations of less than 10 percent in the calibrated current. The tailshock was delivered through electrodes described by Weiss [21] and liberally coated with Redux to maintain optimal electrical contact.

All trials were programmed with BRS equipment according to an intertrial interval averaging $100 \mathrm{sec}$ with a range of $40-160 \mathrm{sec}$. Following this training session, the animals were permitted to recover from paralysis and returned to their home cage. This procedure was repeated for two additional days.

Data analysis. A grass model 7B polygraph was used to record the EKG. Heart signals were recorded from two subdermal 28 ga $5 / 8$ in. hypodermic stainless steel electrodes inserted laterally on the mid-thoracic area. The signals were led into a grass preamplifier (model 7P5-A) and driver amplifier. Heart rate measurements were determined by manually counting the number of heart beats that occurred during the $5 \mathrm{sec}$ periods preceeding the CS onset, during the CS presentation, and following the UCS termination. The conditioned response (CR) measure was defined as the CS-Pre-CS difference score. This was computed by merely subtracting the Pre-CS rate from the CS rate, and thereby allowing HR decelerations to be plotted as a negative function. The unconditioned response (UCR) measurement was also defined as a difference score, but obtained by subtracting the Pre-CS rate from the Post-UCS rate. Accordingly, a positive sign would indicate that shock elevated the $H R$ relative to the $C S$ period. All HR values were converted to beats per minute (BPM) for statistical analysis of variance (ANOVA). 
In order to avoid the risk of superimposing the conditioning trials on a period of EMG recovery from paralysis, the decision was made to omit the presentation of CS-only habituation trials prior to conditioning. Due to this intentional omission, cursory examination of the data revealed an initial, unconditioned, cardiac orienting deceleration that generally habituated within 5 trials. In order to attenuate this resultant biphasic response, the first block of 5 trials in each conditioning session was discarded in the data analysis.

\section{RESULTS AND DISCUSSION}

A $2 \times 2$ (Paired/Unpaired $\times$ Adaptation/Nonadaptation) ANOVA with two repeated measures were conducted on the Pre-CS estimate of basal HR, CS-Pre-CS, and Post UCS-Pre-CS $\mathrm{Hr}$ difference scores. The repeated measures were training sessions and 5 trial blocks.

The mean basal HR (BPM) estimates recorded for the 5 sec Pre-CS period during each training session are shown in Fig. 1. The suggestion that CS-UCS pairings tended to accelerate basal HR is revealed in the significant Paired/ Unpaired main effect, $\mathrm{F}(1,20)=4.688, p<0.05$. Accordingly, it appears that the groups experiencing the contiguous CS-UCS presentation exhibit a higher $(\overline{\mathrm{X}}=434.14)$ level of basal cardiac arousal than the unpaired control groups $(\overline{\mathrm{X}}=414.30)$. The reliable sessions main effect, $F(3,20)=3.550, p<0.05$, reflects a general trend of cardioacceleration with successive training sessions $(\bar{X}=415.78$, 423.38 and 433.50 , for Sessions $1-3$, respectively). A similar trend of cardioacceleration within the training sessions was revealed in a significant Blocks main effect, $\mathbf{F}(5,100)=11.275, p<0.001$. This blocks effect (not shown in Fig. 1) indicates that basal HR was lower during the first 5 trials of each session $(\bar{X}=417.29 \mathrm{bpm})$ than during the last 5 trials $(\overline{\mathrm{N}}=427.60 \mathrm{bpm})$.

Although no Adaptation/Nonadaptation main effect was found, the significant Paired/Unpaired $x$ Adaptation/ Nonadaptation $x$ Session interaction, $F(2,40)=3.609$, $p<0.05$, suggests that the influence of the CS-UCS contingency on basal HR acceleration across Sessions was more pronounced for the curare-adapted animals. No additional effects were observed.

Figure 2 depicts the means CS-Pre-CS HR differences score that occurred across conditioning sessions and blocks of trials. Individual distributions show HR as a function of both the CS-UCS contingency and curare adaptation experience. From the significant Paired/Unpaired, $F(1,20)=$ $29.941, p<0.001$, and Adaptation/Nonadaptation, $F(1,20)$ $=23.021, p<0.001$, main effects, as well as the reliable Paired/Unpaired $\times$ Adaptation/Nonadaptation interaction, $F(1,20)=17.328, p<0.001$, it appears that immobilization facilitates the CS control of HR decelerations produced by the paired CS-UCS presentations. The tendency across all groups for the amplitude of the cardio-deceleration to diminish within each training session (means $=-4.45$ and -2.27 BPM for the first and last blocks of 5 trials) is reflected in the significant Block effect, $F(5,100)=2.327$, $p<0.05$. However, the reliable Paired/Unpaired $\times$ Adaptation/Nonadaptation $\times$ Session interaction, $F(2,40)=$ $11.460, p<0.001$, suggest that the pronounced CS control over HR decelerations improves with additional training sessions. The additional significant Paired/Unpaired $x$ Sessions $\times$ Block, $F(10,200)=2.07, p<0.05$, interaction is not believed to contribute substantially to the interpre-

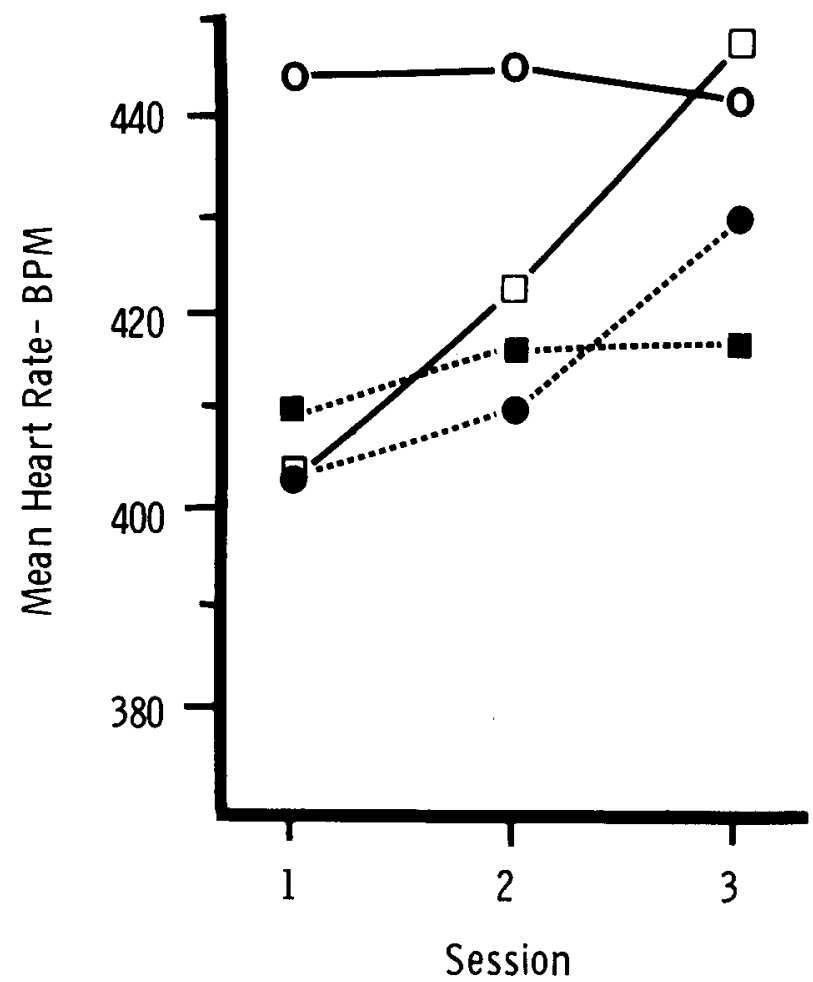

FIG. 1. Mean PreCS basal heart rate in beats per minute (BPM) for each group as a function of three 30-trial conditioning sessions. $\circ \longrightarrow$, PA; $\longrightarrow \longrightarrow$, P-NA; •- - •, UP-A ; - . - UP-NA. (Abbreviations: P-A = Paired-Adapted; P-NA = Paired-Nonadapted; UP-A = Unpaired-Adapted; UP-NA = Unpaired-Nonadapted.)

tation of the data, but generally tends to reflect progressive tendencies for the amplitude of the HR deceleration to diminish across blocks, but increase across sessions as a function of the specific shock and curare immobilization experience. The significance of these latter two triple interaction analyses for the general implications of this study are difficult to assess. No other main effects or interactions were found to be reliable.

The analysis of the Post UCS-Pre-CS HR difference scores were examined only for the groups experiencing the Paired CS-UCS contingency. Accordingly, the $2 \times 3 \times 6$ (Adaptation/Nonadaptation $\times$ Session $\times$ Block) ANOVA with repeated measures on the last two variables revealed no significant main effects. This suggests that the failure to classically condition HR decelerations in the Nonadapted, curarized rat was not due to a disruption of visceral efferent mechanisms involved in conditioned HR activity.

From the results of this experiment, it is apparent that repeated curarization selectively facilitated the HR decelerations in those animals exposed to paired CS-UCS presentations. Although elevated Pre-CS basal HR estimates were also observed for this group, it seems unlikely that the Law of Initial Values could be invoked as a major influence for the conditional deceleration because the Pre-CS HR of the Paired-Nonadapted (P-NA) group was also high in the last training session, but no evidence of conditioning was observed. 


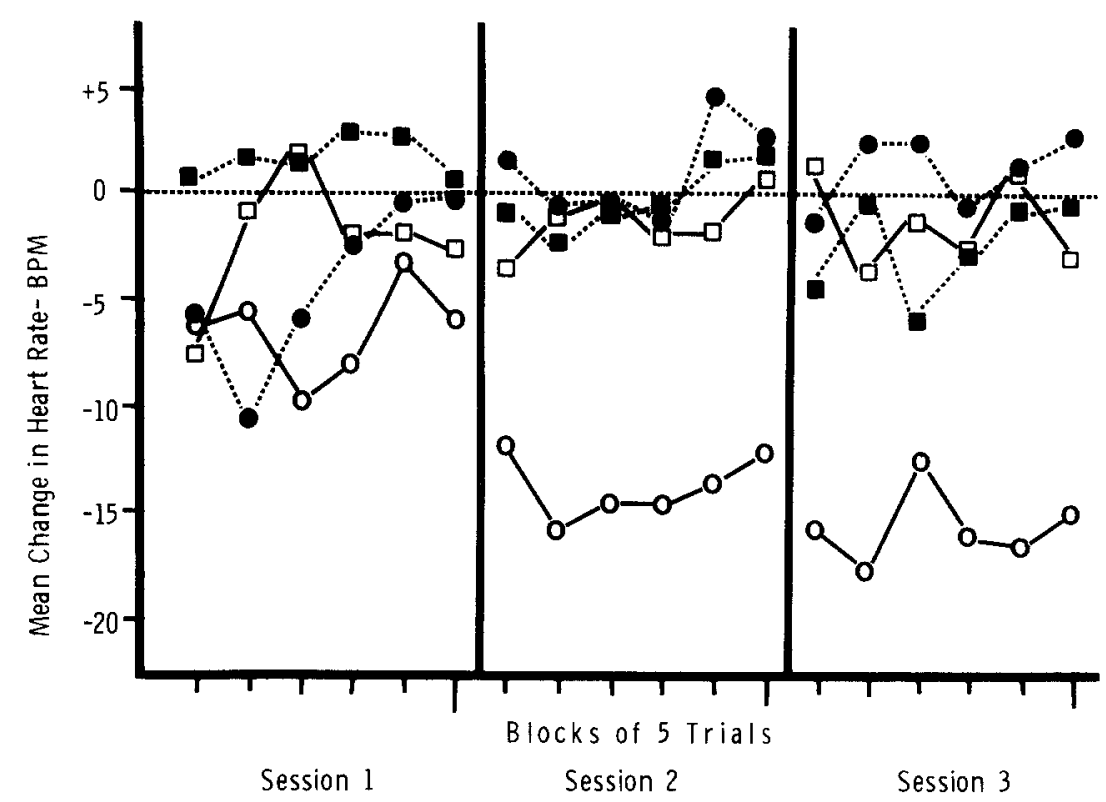

FIG. 2. Mean CS-PreCS heart rate in beats per minute (BPM) for each group as a function of 5-trial blocks of trails during each 30-trial conditioning session. (Legend and abbreviations are the same as in Fig. 1.)

\section{EXPERIMENT 2}

If the curare-adapted animals were recovering faster from neuromuscular paralysis then the pronounced HR CRs might be interpreted as secondary to some peripheral physiological change accompanying skeletal activity [15]. Accordingly, the second experiment was conducted to examine the influence of repeated curare immobilization on tolerance to curare-induced paralysis.

\section{METHOD}

\section{Procedure}

Selection, purchase, and maintenance of the animals were similar to Experiment 1. Twelve rats were randomly assigned to 2 groups, each consisting of 6 animals. The Curare-Adapted (CA) group consisted of 6 rats that had been curarized and artificially respirated in a manner described in the previous two experiments. This immobilization procedure was distributed over 6 consecutive days. For comparison, the remaining 6 animals served as NonCurare Adapted (NCA) controls. These animals were not curare adapted.

The curare immobilization procedure was similar to the procedure used in the previous experiment except for the following: For the last day of curare immobilization for the CA group and for the first (and only) day of curarization for the NCA group, the electromyographic (EMG) activity was recorded instead of HR. The EMG measures were recorded through dual monopolar needle electrodes (Grass Instruments Co.) inserted intramuscularly, and separated by $0.5 \mathrm{~cm}$. Two muscle groups were sampled in each animal. This included the large biceps femoris muscle of the hindlimb and the biceps brachii muscle of the forelimb. EMG potentials were led to Grass Model 7P5-A AC preamplifier set at $10 \mathrm{mv} / \mathrm{cm}$ sensitivity. Recordings were made with the low pass filter set at $75 \mathrm{~Hz} 1 / 2$ amplitude and with the high pass filter set at $3 \mathrm{~Hz} 1 / 2$ amplitude. The signals were integrated electronically by a Grass Model 7-B polygraph integrator channel. Changing EMG potentials were rectified and linearly transduced into positive pen deflections to normalize the response. Continuous records of EMG activity were obtained by maintaining the polygraph paper chart drive at $2.5 \mathrm{~mm} / \mathrm{sec}$ throughout the entire recovery period. The integrator reset values were similar for both EMG recording channels so that changes in EMG from baseline for each muscle group sampled would be reflected by proportional adjustments in the integrator reset values. In this manner, EMG integrator reset values were used as described by Wilson and DiCara [24] as the basis for analyzing between group differences on both the initial level of neuromuscular paralysis as well as course of EMG recovery from paralysis.

Baseline EMG were established $30 \mathrm{~min}$ after curarization to permit maximum immobilization to occur and stabilize. From this point, and throughout the recovery period the EMGs were continuously monitored until the EMG integrator reset value reached 30 percent of its baseline value. The time to reach this criterion was recorded, and the animal was allowed to continue its normal course of recovery from immobilization.

\section{RESULTS AND DISCUSSION}

Group comparisons of the initial integrator reset interval $(\mathrm{cms})$ established $30 \mathrm{~min}$ after curarization and the total time $(\mathrm{min})$ needed to recover to 30 percent baseline paralysis were conducted with 2 -tailed independent $t$-tests. The analysis revealed no evidence that the CA group significantly affected the initial level of curarization. Comparisons between the NCA group (Mean $=24.13 \pm 6.30 \mathrm{cms}$ ) and the CA group $($ Mean $=26.35 \pm 3.86 \mathrm{cms})$ were not statistically 
consistent $(t=0.302)$. Thus, it would seem that the initial level of neuromuscular paralysis was similar across both groups.

A similar absence of a treatment effect was observed for the recovery time $(\mathrm{min})$ from paralysis. $t$-Tests showed that the mean recovery times for the NCA group (136.17 \pm $16.47 \mathrm{~min})$ and the $\mathrm{CA}$ group $(153.17 \pm 10.73 \mathrm{~min})$ were not reliably different $(t=0.858)$. In fact, the tendency was for the repeated curare immobilization to lower, rather than elevate, the threshold for neuromuscular paralysis. In view of this data, there appears to be no convincing evidence that repeated curarization, which were shown to influence acquisition of a Pavlovian conditioned HR deceleration, had any systematic effect on the recovery from dTC-induced neuromuscular paralysis.

\section{GENERAL DISCUSSION}

These results indicate that immobilization-induced stress, rather than inadequate respiration or pharmacological consequences of curarization may underlie certain difficulties experienced with the curarized rat. Accordingly, adaptation to this stress or perhaps use of a stress-resistent strain of rat, would seem to be a requisite for stimulus control of HR changes during paralysis. The fact that traditional ventilation and drug-dose parameters were used in the present experiment also emphasizes that assessing the interaction of stress physiology and cardiovascular activity may be more desirable than searching for the suitable combination of neuromuscular drug-dose and respiration system.

The characteristics of the conditioned HR response were generally consistent with observations that Pavlovian HR CRs in the physically restrained rat takes the form of cardiodecelerations [19]. The tendency for the response to increase then diminish within each conditioning session confirms Holdstock and Schwartzbaum's [11] find ing that, although the average strength of the CR increases across sessions, the rate of diminution of $C R$ strength within sessions also increases. The possibility that these diminutions reflect sympathetic competition or conditioned inhibition of delay $[12,13]$ is difficult to assess from this data. In contrast, the amplitude of the HR CR for the Paired-Adapted animals was lower than reported for the physically restrained rat $[5,6,20]$. However, the fact that performance was improving across the 3 conditioning sessions suggests that additional training would have produced greater cardiodecelerations.

The different basal HRs for the Paired and Unpaired animals, as well as the absence of conditioning for the Paired-Nonadapted group is difficult to interpret but is not unprecedented. Holdstock and Schwartzbaum [11] observed that Pre-CS basal HR estimates accelerated within one Pavlovian conditioning in the noncurarized rat. Moreover, other studies have shown that basal HRs progressively increase over consecutive conditioning sessions for rats experiencing paired CS-UCS presentations while curarized [16] or physically restrained [17]. The fact that the P-NA group exhibited no HR CR over the 3 conditioning sessions confirms Ray's [16] finding for 3 of 4 sessions using a similar conditioning and curarization procedure. These animals apparently benefit neither from over 100 total paired CS-UCS presentations or the curare exposure accumulated over the 3 sessions. However, the pronounced HR decelerations demonstrated for the P-A animals implies that conditioning would have been observed for the P-NA group if conditioning had been extended beyond 3 sessions. Unfortunately, the present experiment was not designed for this kind of analysis. However, Ray's [16] data show that the HR CR on his fourth conditioning session were strikingly similar to those found for the P-A group of the present study on the first conditioning session following curare adaptation. Accordingly, it seems reasonably to speculate that extended conditioning of the P-NA group would have produced stable HR CRs approaching those in magnitude of the P-A group.

It seems clear that the processes underlying the adaptation-induced facilitation of $H R$ conditioning in the curarized rat are independent of differences in basal HR or sensitization. The absence of any evidence of drug tolerance over repeated curare paralysis also strongly suggest that the HR CRs were not reflexive cardiovascular changes reflecting merely differences in the reinstatement of skeletal-motor control. In general, then, it appears that the consequences of neuromuscular paralysis in curare-adapted rats is considerably different from what might be anticipated from the recent autonomic conditioning literature using the curarized preparation. Accordingly, curarization, like conventional physical restraining procedures, may provide a variable technique for assessing the influence of stress physiology on basal and conditioned cardiovascular activity.

\section{REFERENCES}

1. Black, A. H. Operant conditioning of heart rate under curare. Technical Report No. 12, Department of Psychology, McMaster University, Hamilton, Ontario, 1967.

2. DiCara, L. V. Analysis of arterial blood gases in the curarized, artificially respirated rat. Behav. Res. Meth. Inst. 2: 67-69, 1970.

3. DiCara, L. V. Some critical methodological variables involved in instrumental visceral learning. In: Contemporary Trends in Cardiovascular Psycho-physiology - Current Issues in Response Mechanisms, Biofeedback and Methodology, edited by P. A. Obrist, A. H. Black, J. Brener and L. V. DiCara. Chicago: Aldine-A therton, 1974, pp. 276-294.

4. Dworkin, B. R. An effort to replicate visceral learning in curarized rats. Unpublished dissertation, The Rockefeller University, 1973.
5. Fitzgerald, R. D., R. M. Vardaris and J. S. Brown. Classical conditioning of heart rate deceleration in the rat with continuous and partial reinforcement. Psychon. Sci. 6: 437-438, 1966.

6. Fitzgerald, R. D., G. K. Martin and J. H. O'Brian. Influence of vagal activity on classically conditioned heart rate in rats. $J$. comp. physiol. Psychol. 83: 485-491, 1973.

7. Gaebelein, C. J., J. L. Howard, R. A. Galsoy and P. A. Obrist. Classical aversive conditioning under different neuromuscular blocking agents in cats. Paper presented at the 12th Annual Meeting of the Society for Psychophysiological Research, Boston, Massachusetts, 1972. 
8. Galosy, R. A., J. L. Howard, C. J. Gaebelein and P. A. Obrist. Neuromuscular blocking drugs and the heart rate response to direct preganglionic stimulation of the sympathetics and parasympathetics in the cat. Paper presented at the 12 th Annual Meeting of the Society for Psychophysiological Research, Boston, Massachusetts, 1972.

9. Hahn, W. W. The learning of autonomic responses by curarized animals. In: Contemporary Trends in Cardiovascular Psychophysiology - Current Issues in Response Mechanisms, Biofeedback and Methodology, edited by P. A. Obrist, A. H. Black, J. Brenner and L. V. DiCara. Chicago: Aldine-Atherton, 1974, pp. $295-311$

10. Hahn, W. W. and J. Slaughter. Heart rate responses of the curarized rat. Psychophysiology 7: 429 435, 1970.

11. Holdstock, T. and J. Schwartzbaum. Classical conditioning of heart rate and GSR in the rat. Psychophysiology 2: 25-38, 1965 .

12. Kimmel, H. D. Inhibition of the unconditioned response in classical conditioning. Psychol. Rev. 73: 232-240, 1966.

13. Kimmel, H. D. Essential events in the acquisition of classical conditioning. Cond. Reflex. 5: 156-164, 1970.

14. Miller, N. E. and B. R. Dworkin. Visceral learning: Recent difficulties with curarized rats and significant problems for human research. In: Contemporary Trends in Cardiovascular Psychophysiology - Current Issues in Response Mechanisms, Biofeedback and Methodology, edited by P. A. Obrist, A. H. Black, J. Brener and L. V. DiCara. Chicago: Aldine-Atherton, 1974, pp. 312-331.
15. Obrist, P. A., R. A. Webb, J. R. Sutterer and J. D. Howard. The cardiac-somatic relationship: Some reformulations. Psycho physiology 6: 569-587, 1970 .

16. Ray, R. Classical condition of heart rate in restrained and curarized rats. Unpublished dissertation. University of Tennessee, 1969.

17. Ray, R. Use of conditional reflex to assess the temporal characteristics of curarization effects on heart rate responding. Cond. Reflex. 7: 19-32, 1972.

18. Schwartz, M. L. and W. W. Hahn. Physiological adjustment of animals paralyzed by d-tubocurarine chloride. Paper presented at the 82nd Annual Meeting of the Americal Psychological Association, New Orleans, Louisiana, 1974.

19. Teyler, T. J. Effects of restraint on heart rate conditioning in rats as a function of US location. J. comp. physiol. Psychol. 77: $31-37,1971$

20. Vardaris, R. M. Partial reinforcement and extinction of heart rate deceleration in rats with the US interpolated on nonreinforcement trials. Learn. Motiv. 2: 280--288, 1971.

21. Weiss, J. M. A tail electrode for unrestrained rats. J. exp. Analysis Behav. 10: 85-86, 1967.

22. Weiss, J. M. Somatic effects of predictable and unpredictable shock. Psychosom. Med. 32: $397-408,1970$.

23. Wilder, J. The law of initial values in neurology and psychiatry: Facts and problems. J. nerv. ment. Dis. 125: $73 \cdots 86,1957$.

24. Wilson, J. R. and L. V. DiCara. Influence of neuromuscular blocking drugs on recovery of skeletal electromyographic activity in the rat. Psychophysiology in press. 